\title{
Microscale scavenging of pentachlorophenol in water using amine and tripolyphosphate-grafted SBA-15 silica: Batch and modeling studies
}

\author{
Paul N. Diagboya a, b, *, Bamidele I. Olu-Owolabi a, Kayode O. Adebowale a \\ a Department of Chemistry, University of Ibadan, Ibadan, Nigeria \\ ${ }^{\mathrm{b}}$ National Center for Nanoscience and Technology, Beijing 100190, China
}

\section{A R T I C L E I N F O}

\section{Article history:}

Received 27 January 2014

Received in revised form

12 April 2014

Accepted 22 April 2014

Available online

\section{Keywords:}

Functionalization

Mesoporous silica SBA-15

Pentachlorophenol

Tripolyphosphate

Adsorption

\begin{abstract}
A B S T R A C T
Mesoporous silica SBA-15 meets most criteria for selection of water treatment adsorbents such as high specific surface area, large pore-size, chemical inertness, repertory of surface functional groups, good thermal stability, selectivity, regenerability, and low cost of manufacture. However, its use for water treatment is still largely unexplored. SBA-15 and its functionalized derivatives of aminopropyltriethoxysilane (SA) and tripolyphosphate (ST) were synthesized, characterized, and used to investigate pentachlorophenol (PCP) removal from aqueous solutions. Functionalization improved SBA15 capacity for PCP removal from solution in accordance with the trend SBA- $15<$ ST $<$ SA. Sorption rate experiments data fit the Lagergren pseudo-second order kinetics model. Intra-particle diffusion indicated that the sorption is controlled by two mechanisms: intra-particle and equilibrium diffusion. Adsorptive pore-filling and electrostatic interactions were implicated in the removal of PCP from solution. Electrostatic interaction led to $\geq 75 \%$ increase in sorption upon functionalization. The equilibrium sorption data of the PCP on these mesoporous materials fits the Freundlich isotherm. Desorption hysteresis was low for the pristine SBA-15, but the functionalized SBA-15 materials showed higher hysteresis. The results imply that functionalized SBA-15 sorbents are promising materials for microscale scavenging of PCP in solution.
\end{abstract}

() 2014 Elsevier Ltd. All rights reserved.

\section{Introduction}

Pentachlorophenol (PCP) and its water soluble salt, sodium pentachlorophenate, are commercially produced synthetic organochlorine compounds used primarily as preservatives for wood and wood products, and secondarily as herbicides, insecticides, fungicides, molluscicides, and bactericides. PCP persists in the environment for a long and is well known for its toxicity to biota (Abdel Salam and Burk, 2009; EPA, 1980). Before 1984, it was one of the most widely used biocides, and this has led to its prevalence in the environment. This has exposed humans to significant amounts of PCP especially through water sources (Fisher, 1991). Detectable PCP levels have been found in most people living in industrialized societies, probably as a result of food chain exposure to PCP in the environment (Eisler, 1989; Fisher, 1991). PCP is sparingly soluble in water but the removal of this toxic compound from aqueous systems represents a problem especially at low concentrations (Abdel

\footnotetext{
* Corresponding author. Department of Chemistry, University of Ibadan, Ibadan, Nigeria. Tel.: +2348038052553.

E-mail address: pauldn2@yahoo.com (P.N. Diagboya).
}

Salam and Burk, 2009); hence, there is need to develop efficient removal methods.

The conventional treatment methods for wastewater include reverse osmosis, electrochemical treatment, solvent extraction, and adsorption on activated carbon, biological treatment (Olu-Owolabi et al., 2012). Several techno-economic considerations, such as efficiency and cost, plague these techniques. Of these techniques, adsorption is the most promising for the removal of solution PCP because via the adsorption-desorption processes, the water and sorbent can be recycled. Thus, there is need to develop viable sorbents as well as understand the adsorption characteristics of PCP from aqueous solutions.

There is a growing interest in the adsorption chemistry of ordered meso-porous silica (SBA-15) in relation to organic pollutants (Bernardoni and Fadeev, 2011). This interest stem from the fact that SBA-15 meets most criteria for selection of adsorbents: sorption capacity, selectivity, regenerability, kinetics, compatibility, and cost. It has such remarkable properties as high specific surface area, large pore-size, chemical inertness, abundant repertory of surface functional groups, good thermal stability, and low cost of manufacture, as well as the recent successful applications of this material in adsorption (Bui et al., 2011; Momcilovic et al., 2013). SBA-15 are hexagonally 
ordered mesoporous molecular sieves which can be synthesized to include a wide variety of functionally active chemical compositions by exploiting the structure-directing functions of electrostatic, hydrogenbonding, and van-der Waals interactions associated with amphiphilic molecules. Sulfonic acid functionalized SBA-15 (Margolese et al., 2000), carboxylate functionalized SBA-15 (Bruzzoniti et al., 2007) and amine functionalized SBA-15 (Wang et al., 2004) have been synthesized this way. Typically, these materials are synthesized under conditions where silica-surfactant self-assembly occurs simultaneously with condensation of the inorganic species, yielding mesoporous ordered composites. In general, the organic functionality can be introduced by chemical attachment onto the silanol groups in the mesoporous silica or by co-condensation during the template-directed synthesis of the mesoporous material, as well as post synthetic modification of SBA-15. Pure and functionalized SBA-15 have been used for adsorption of both organics and inorganics (Choi et al., 2011; Corriu et al., 2004; Dou et al., 2011; Katiyar et al., 2006). However, no study till date has investigated the ability of SBA-15 and its functionalized derivatives for the sorption of PCP.

The aim of this study was to synthesize and functionalize SBA15 with amine and tripolyphosphate groups, and apply the functionalized SBA-15 sorbents for the removal of PCP from aqueous solution. The sorptions data were explained using kinetics and adsorption isotherm models.

\section{Materials and methods}

\subsection{Synthesis of pristine and functionalized SBA-15}

SBA-15 was synthesized using the modified methods of Zhao et al. (1998) and Wang et al. (2004). Triblock copolymer Pluronic $\mathrm{P} 123\left(\mathrm{EO}_{20} \mathrm{PO}_{70} \mathrm{EO}_{20}\right)$ was used as structure-directing agent, and tetraethyl orthosilicate (TEOS) as substrate. In a typical synthesis, TEOS $(2.10 \mathrm{~g})$ was introduced into aqueous $\mathrm{HCl}(30 \mathrm{~mL} ; 2 \mathrm{M} \mathrm{HCl})$ solution containing Pluronic P123 (1.00 g) and hydrolyzed for $1 \mathrm{~h}$ while stirring at $40{ }^{\circ} \mathrm{C}$ followed by addition of $7.5 \mathrm{~mL}$ Millipore $\mathrm{H}_{2} \mathrm{O}$. The resultant mixture was stirred at $40^{\circ} \mathrm{C}$ for $20 \mathrm{~h}$ followed by aging for $24 \mathrm{~h}$ at $85{ }^{\circ} \mathrm{C}$ under static conditions. The solid crystals were obtained by vacuum filtration and the template (pluronic P123) was removed by washing using the $1 x$ half batch system proposed by Thielemann et al. (2011) by alternating ethanol, water, and ethanol under pressure. Finally, the material was dried at room temperature.

The amine functionalized SBA-15 (SA) was synthesized using Pluronic P123 as template, TEOS and 3-aminopropyltriethoxysilane (APS) as substrates. In the synthetic process, TEOS (2.10 g) was introduced to $25 \mathrm{~mL} 2 \mathrm{M} \mathrm{HCl}$ solution containing $1.00 \mathrm{~g}$ Pluronic P123 and hydrolyzed for $1 \mathrm{~h}$ under stirring at $40^{\circ} \mathrm{C}$. Then APS ( $\left.1.10 \mathrm{~g}\right)$ was added into the mixture slowly followed by $7.5 \mathrm{~mL}$ of Millipore $\mathrm{H}_{2} \mathrm{O}$.

The molar composition of the mixture was kept at:

$$
(1-x) \text { TOES: } x \text { APS: } 0.017 \text { P123: } 6.1 \mathrm{HCl}: 169.4 \mathrm{H}_{2} \mathrm{O}
$$

where $x$ is equal to $0.1 \mathrm{M}$ (10\% of proportion of Si in the APS). The resultant mixture was incubated, washed, and filtered as above.

Synthesis of tripolyphosphate functionalized SBA-15 (ST) was done by firstly synthesizing SA as above. Before the $24 \mathrm{~h}$ aging, $30 \mathrm{~mL}$ of $0.25 \mathrm{M}$ NaTPP solution was added to the synthesis mixture and then aged for $24 \mathrm{~h}$ at $85^{\circ} \mathrm{C}$ under static condition. The filtration, template removal and drying are same as above.

\subsection{Characterization of synthesized SBA-15 sorbents}

The surface area and porosity were measured using Micromeritics ASAP 2020M + C accelerated surface area and porosimetry analyzer. The samples were degassed at $80{ }^{\circ} \mathrm{C}$ and the nitrogen adsorption-desorption isotherms obtained were evaluated to give the Brunauer-Emmett-Teller (BET) specific surface area and pore volume. Infra-red (IR) spectra have been obtained using a Spectrum-1 FTIR spectrometer (Perkin-Elmer instruments Co. Ltd, USA) in the scanning frequency of $4500-450 \mathrm{~cm}^{-1}$. Scanning electron microscope (SEM) images were obtained using a Hitachi S4800 microscope (Hitachi Ltd., Japan) with an accelerating voltage of $6.0 \mathrm{kV}$ and equipped with a Horiba energy dispersive $X$-ray spectrometer. Thermo-gravimetric analysis (TGA) was carried out on the various powdery samples using Pyris Diamond thermogravimetric-differential thermal analyzer by heating the samples at $5{ }^{\circ} \mathrm{C} \mathrm{min}^{-1}$ to $800{ }^{\circ} \mathrm{C}$ in nitrogen atmosphere.

\subsection{PCP sorption study}

Stock PCP solution ( $500 \mathrm{mg} \mathrm{L}^{-1}$ ) was prepared by dissolving the appropriate mass of solid PCP in 10\% ethanol:water and stored in the refrigerator at $4{ }^{\circ} \mathrm{C}$ to be used within 14 days of preparation. Working solutions were prepared from the stock in ethanol/ deionized water $(10 \% \mathrm{v} / \mathrm{v})$ using $0.005 \mathrm{M} \mathrm{CaCl}_{2}$ and $0.01 \mathrm{M} \mathrm{NaN}_{3}$ as background electrolyte and biocide to eliminate microbial degradation, respectively. During the sorption study, mass of sorbent to PCP concentration ratios were carefully chosen to ensure that between 10 and $90 \%$ of the PCP in solution were removed. The loss of PCP by photochemical decomposition, volatilization, and sorption on the brown glass vials were compensated for by the use of blanks, and were found to be negligible.

Sorption experiments were done on the SBA-15 sorbents by investigating the effects of incubation time, $\mathrm{pH}$, equilibrium, and desorption. These experiments were done using $40 \mathrm{mg}$ of the sorbents in $10 \mathrm{~mL}$ PCP solution in brown vials sealed tightly with Teflon lined screw caps and covered with aluminum foil to minimize possible losses by photochemical decomposition.

The effect of time was done using vials which were equilibrated in the dark by shaking at $100 \mathrm{rpm}$ in a temperature $\left(25^{\circ} \mathrm{C}\right)$ controlled shaker at various time intervals $(60-2880 \mathrm{~min})$. The effects of $\mathrm{pH}$ and PCP concentration on sorption were studied using $40 \mathrm{mg}$ of the sorbents, $10 \mathrm{~mL}$ PCP solution, at $25^{\circ} \mathrm{C}$, incubation time of $1440 \mathrm{~min}$, and shaking speed of $100 \mathrm{rpm}$. For the effect of $\mathrm{pH}$, the solution pH was varied from 3 to 9; while for effect of concentration, concentrations were varied between 10,000 and $40,000 \mu \mathrm{g} \mathrm{L}^{-1}$ at $\mathrm{pH}$ of 6.5. After each experiment, the vials were centrifuged and the amount of PCP in solution determined. Desorption experiments were immediately carried out after the effect of concentration experiments by carefully decanting the aqueous solution in each centrifuged vial and replacing with the same amount of background solution containing no PCP. The vials were again equilibrated at the respective temperatures for $1440 \mathrm{~min}$, centrifuged, and PCP in solution determined. All experiments were carried out in duplicate. The vial centrifugations were done at $4000 \mathrm{rpm}$ for $15 \mathrm{~min}$, and the PCP determined using the Perkin-Elmer Lamda 950 high performance UV-vis-NIR spectrophotometer and $1 \mathrm{~cm}$ path length quartz cells. The peak at $320 \mathrm{~nm}$ was used to monitor the PCP sorption.

\subsection{Data treatment}

The amounts of PCP sorbed were calculated using Equation (1):

$q_{e}=\frac{\left(C_{O}-C_{e}\right) V}{M}$

where $\left(C_{o}\right)$ and $\left(C_{e}\right)$ are initial and final PCP concentrations $\left(\mu \mathrm{g} \mathrm{L}^{-1}\right)$ in solutions, respectively, $q_{e}, V$ and $M$ are the amount of PCP sorbed 
$(\mu \mathrm{g} / \mathrm{g})$, volume of the solution $(\mathrm{mL})$ and mass $(\mathrm{g})$ of sorbent, respectively.

Two adsorption models - Langmuir (1916) and Freundlich (1906), in their linear forms were employed in describing the adsorption process. The Lagergren (1898) pseudo-first order and pseudo-second order models, the Elovich (Olu-Owolabi et al., 2014), and the Weber and Morris (1963) intra-particle diffusion (IPD) models were used to describe the adsorption data (Supporting material).

\section{Results and discussions}

\subsection{Synthesis and characterization of the SBA-15 sorbents}

The structure of SBA-15, the schematics of the synthesis of SA and ST, as well as the proposed interaction of tripolyphosphate with SA within a mesopore of SBA-15 are shown in Fig. 1(a-d). SBA-15 or its derivatives can be synthesized over a narrow range of reaction mixture compositions and conditions. Thus, care was taken to avoid unintended results during the synthesis because the use of concentrations of Pluronic P123 higher than 6 weight \% yields only silica gel or produces no precipitation of silica, whereas concentrations below 0.5 weight \% result in only amorphous silica; again, at room temperature or temperatures lower than $40{ }^{\circ} \mathrm{C}$, only amorphous silica powder or poorly ordered patterns are obtained, whereas at higher temperatures, silica gel is produced; at $\mathrm{pH}$ values from 2 to 6 , above the isoelectric point of silica $(\mathrm{pH}-2)$, no precipitation of silica gel occurs, at neutral $\mathrm{pH}$; only disordered or amorphous silica is obtained (Zhao et al., 1998). Hence, SBA-15 sorbents were synthesized using 5\% weight Pluronic P123 copolymer at $40{ }^{\circ} \mathrm{C}$ at $\mathrm{pH} 1$ following the combined methods of Zhao et al. (1998) and (Wang et al., 2004). Calleja et al. (2011) have
Table 1

Characterization data of the SBA-15, SA, and ST mesostructures.

\begin{tabular}{lllll}
\hline Sample & \multicolumn{2}{l}{ Surface area $\mathrm{m}^{2} \mathrm{~g}^{-1}$} & Pore volume $\mathrm{cm}^{3} \mathrm{~g}^{-1}$ & Pore diameter $\mathrm{nm}$ \\
\cline { 2 - 4 } & BET & Langmuir & & \\
\hline SBA-15 & 539.19 & 757.68 & 0.85 & 6.44 \\
SA & 125.18 & 175.33 & 0.10 & 4.81 \\
ST & 248.62 & 350.86 & 0.49 & 7.72 \\
\hline
\end{tabular}

shown that primary amines give products of higher surface areas than the secondary or tertiary amines, and that low temperature (room temperature) is better for SBA-15 drying; hence the use of APS and room temperature for drying.

The shapes of the isotherm curves obtained from the nitrogen adsorption-desorption of SBA-15, SA, and ST (See SI, Figure S1) suggested a type IV Brunauer-Emmett-Teller (BET) isotherm, an indication of the uniformity and stability of the mesoporous structure of these materials even after the functionalization. The stability varies according to the trend: SBA-15 $>$ ST $>$ SA. Significant desorption hysteresis were observed for SBA-15 and ST between $0.5<\mathrm{P} /$ Po $<0.9$, but not the SA. Upon functionalization of the SBA-15 framework wall, significant decreases in the surface areas were observed (Table 1) due to the attachment of the APS on the framework mesopores. The surface areas of SBA-15, SA, and ST are 539.19, 125.18 , and $248.62 \mathrm{~m}^{2} \mathrm{~g}^{-1}$, respectively. Similar size reductions were also observed for the pore volumes of ST and SA (Table 1; Figure S1). However, the pore diameter of ST increased while that of SA decreased. These observed changes are indications of the successful modifications of SBA-15 mesopores as shown in Fig. 1. Similar results have been observed by Xu et al. (2011) and Mureseanu et al. (2008).

The infrared spectra of the pure and functionalized SBA-15 mesoporous silicas are shown in Fig. 2a (black spectra). The pure
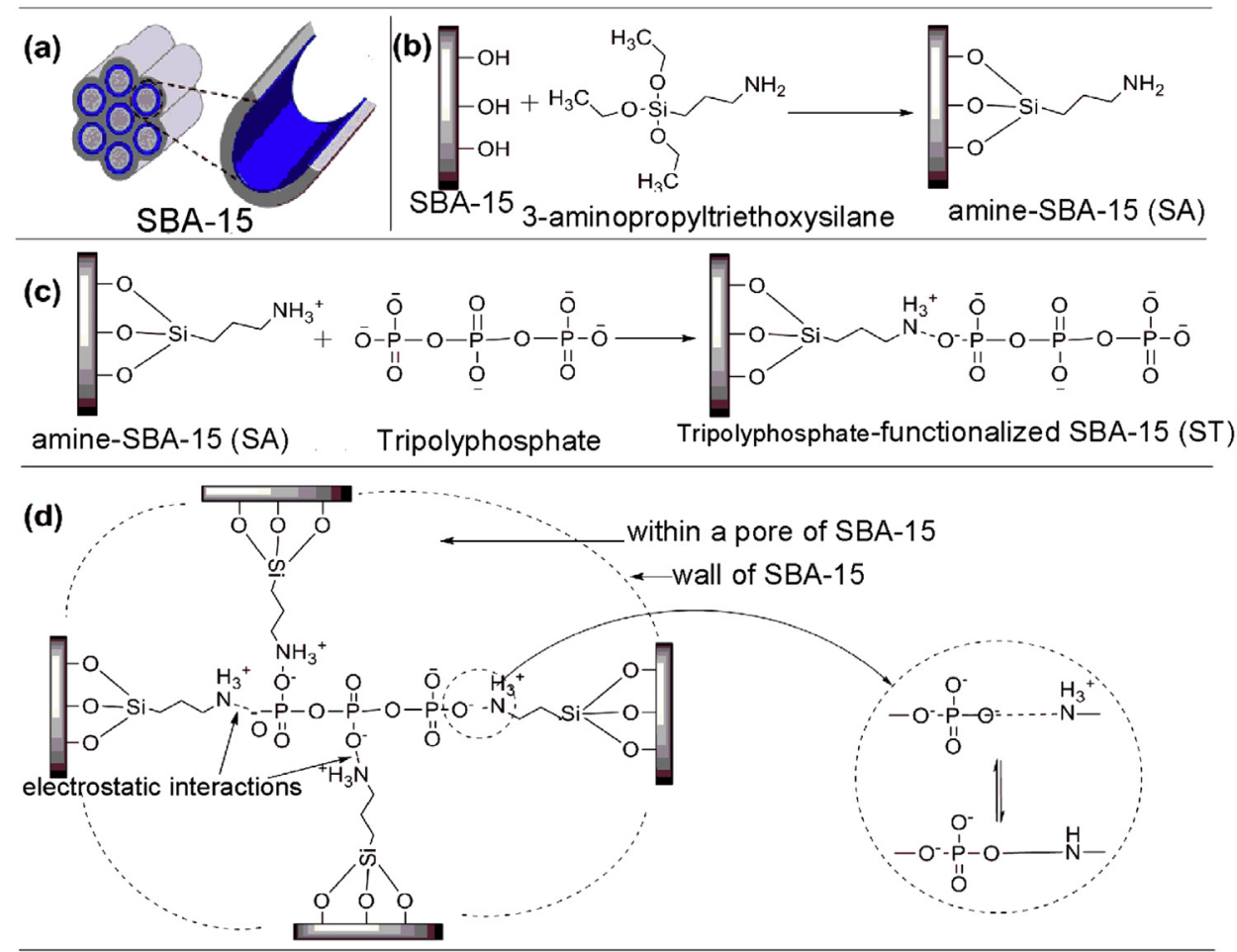

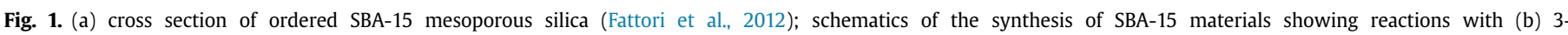

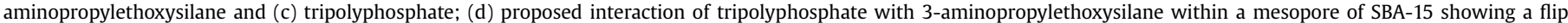

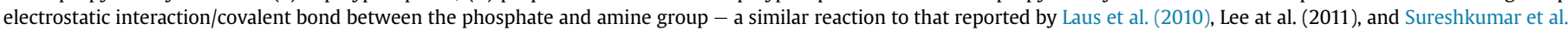
(2010) but with little modification. 

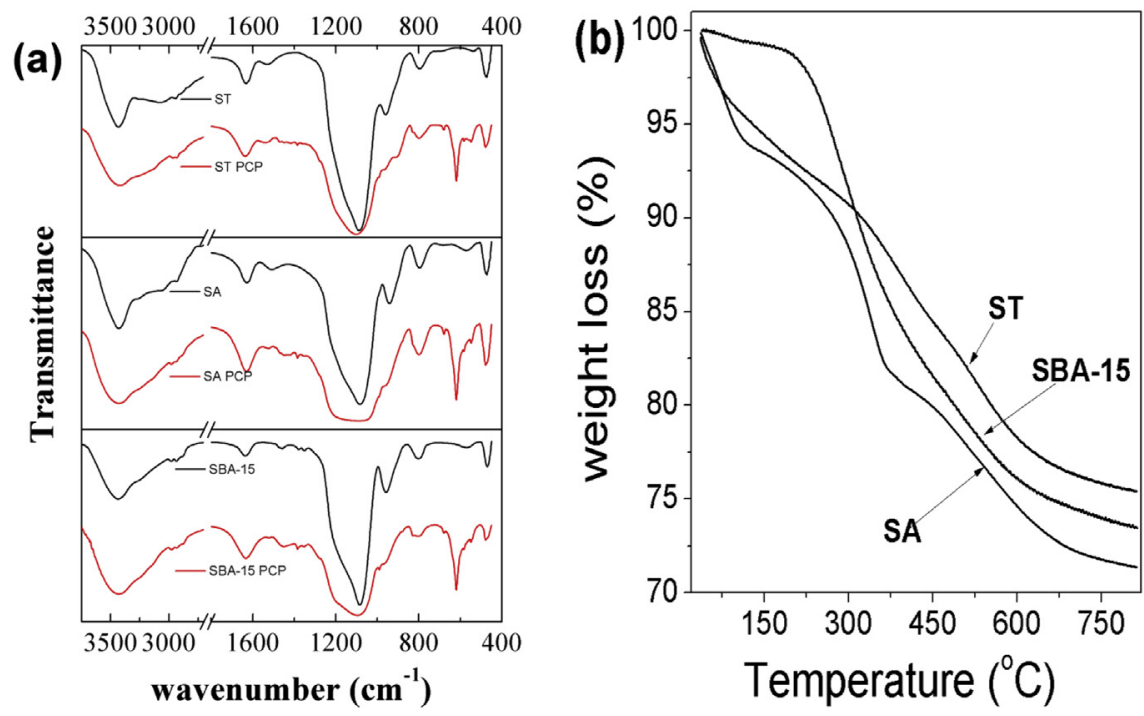

Fig. 2. (a) IR spectra of SBA-15, SA, and ST (the black and red curves are the spectra before and after sorption, respectively); (b) thermo-gravimetric analysis spectra.

SBA-15 showed a typical broad peak around $3450 \mathrm{~cm}^{-1}$ associated with the presence of silanol groups. After functionalization with the amino-propyl group of the APS, the width of this peak in the new materials (SA and ST) decreased with corresponding increase in intensity in both new materials. This is typical of the immobilized amino-propyl group on the framework of the SBA-15 (Bui et al., 2011). The functionalized SBA-15 materials also showed additional peak around the $1560 \mathrm{~cm}^{-1}$ which is indicative of the amide-I structure from the condensed amino-propyl-tri-ethoxy-silane. These peaks suggested successful reaction between the hydroxyl groups on the silica framework and the ethoxy groups of the amino-propyl-tri-ethoxy-silane substrate. The peaks observed between 2800 and $3000 \mathrm{~cm}^{-1}$ were attributed to both symmetric and asymmetric stretching vibrations of the $-\mathrm{CH}_{3}$ and $-\mathrm{CH}_{2}$ groups of the APS (Mureseanu et al., 2008). On functionalization with the tripolyphosphate, the intensity of the peak around $1070 \mathrm{~cm}^{-1}$ increased and a new peak appeared at $953 \mathrm{~cm}^{-1}$. These peaks are associated with phosphate and phosphoramide groups, and it suggests reaction between $-\mathrm{P}_{3} \mathrm{O}_{10}^{5-}$ and $-\mathrm{NH}_{3}^{+}$of the APS group. Similar observations have been reported by Panda et al. (2003), Parikh and Chorover (2006), and Lee et al. (2001).

The infrared spectra of the SBA-15 sorbents after PCP sorptions are also shown in Fig. 2a (red spectra). Significant changes where observed in the intensity and broadness of the initial peaks observed in the pure and functionalized SBA-15 materials; these are clear indications of the adsorption of PCP on these sorbent materials.

Fig. 2b shows the thermogravimetric analysis (TGA) of SBA-15, SA, and ST. The quantity of organic and inorganic functional groups incorporated into the mesoporous SBA-15 was estimated from this analysis. The weight losses at temperatures below $150{ }^{\circ} \mathrm{C}$ have been attributed to removal of the physisorbed water. Weight losses below this temperature indicated that the functionalized silica materials adsorbed more water than the pristine silica, and SA > ST. The large weight losses from 200 to $600{ }^{\circ} \mathrm{C}$ have been linked to the decomposition of the bridged organic moieties which are anchored on the SBA-15 surface. The percentage of materials left at $700{ }^{\circ} \mathrm{C}$ shows that ST has better thermal stability than SA. TGA results demonstrated that both new materials have a good degree of surface functionalization. Dou et al. (2011) and Li et al. (2007) have reported similar findings for SBA-15 materials.
The scanning electron microscope (SEM) images of SBA-15, SA, and ST are shown in Fig. 3. The morphology of these materials clearly exhibited hexagonal arrangements of relatively uniform short particles as shown in Fig. 1a. The SEM images of the functionalized SBA-15 materials exhibited similar meso-structure type, indicating no change in the morphology of SBA-15 after functionalization with the amino-propyl-tri-ethoxy-silane and tripolyphosphate. Thus, the ordered meso-structure of the mesoporous SBA-15 is preserved after functionalization of the surface.

\subsection{Sorption of PCP by SBA-15 materials}

Determining how fast a pollutant is removed from solution by a sorbent is an important way of accessing the efficiency of the sorbent for removal of that pollutant from aqueous solution. This is done by determining the time it takes for $1.0 \mathrm{~g}$ of an adsorbent to be saturated by a sorbate. Hence, the effect of time on the sorption of PCP from solution using the synthesized SBA-15, SA, and ST was determined over a 2880 min period. Data generated were used to study the adsorption kinetics. The effect of time on PCP sorption is shown in Fig. 4a. The steep portion of the curve represents PCP sorption prior to equilibrium where the rate of sorption exceeded the rate of desorption. At equilibrium, the rate of sorption and desorption is insignificant and the curve becomes nearly horizontal. Equilibrium was attained for the adsorbents at $720 \mathrm{~min}$. At this point, 5.8\% (579.8 $\left.\mu \mathrm{g} \mathrm{g}^{-1}\right), 32.8 \%\left(3279.6 \mu \mathrm{g} \mathrm{g}^{-1}\right)$, and $24.6 \%$ $\left(2462.8 \mu \mathrm{g} \mathrm{g}^{-1}\right)$ of PCP had been removed from solution by SBA-15, SA, and ST, respectively. The low PCP sorption on SBA-15 was attributed to the nature of its surface (Fig. 1a) which has no active functional groups to interact with the predominantly negatively charged PCP (pentachlorophenolate) species in solution (Fig. 5right) which is its ionization state at the solution $\mathrm{pH}(6.5 \pm 0.2)$ ( $\mathrm{Li}$ et al., 2009). Thus, the two mechanisms thought to be responsible for the removal of PCP by SBA-15 are via very weak interactions with SBA-15 external wall surfaces, which accounts for very little PCP removal, and the filling of SBA-15 mesopores by charged PCP species in solution which was significant for SBA-15. The situation is, however, different for the amine-functionalized SBA-15 (SA) with a positively charged amine group $\left(-\mathrm{NH}_{3}^{+}\right)$at the sorption solution $\mathrm{pH}$. Electrostatic interaction between the positively 

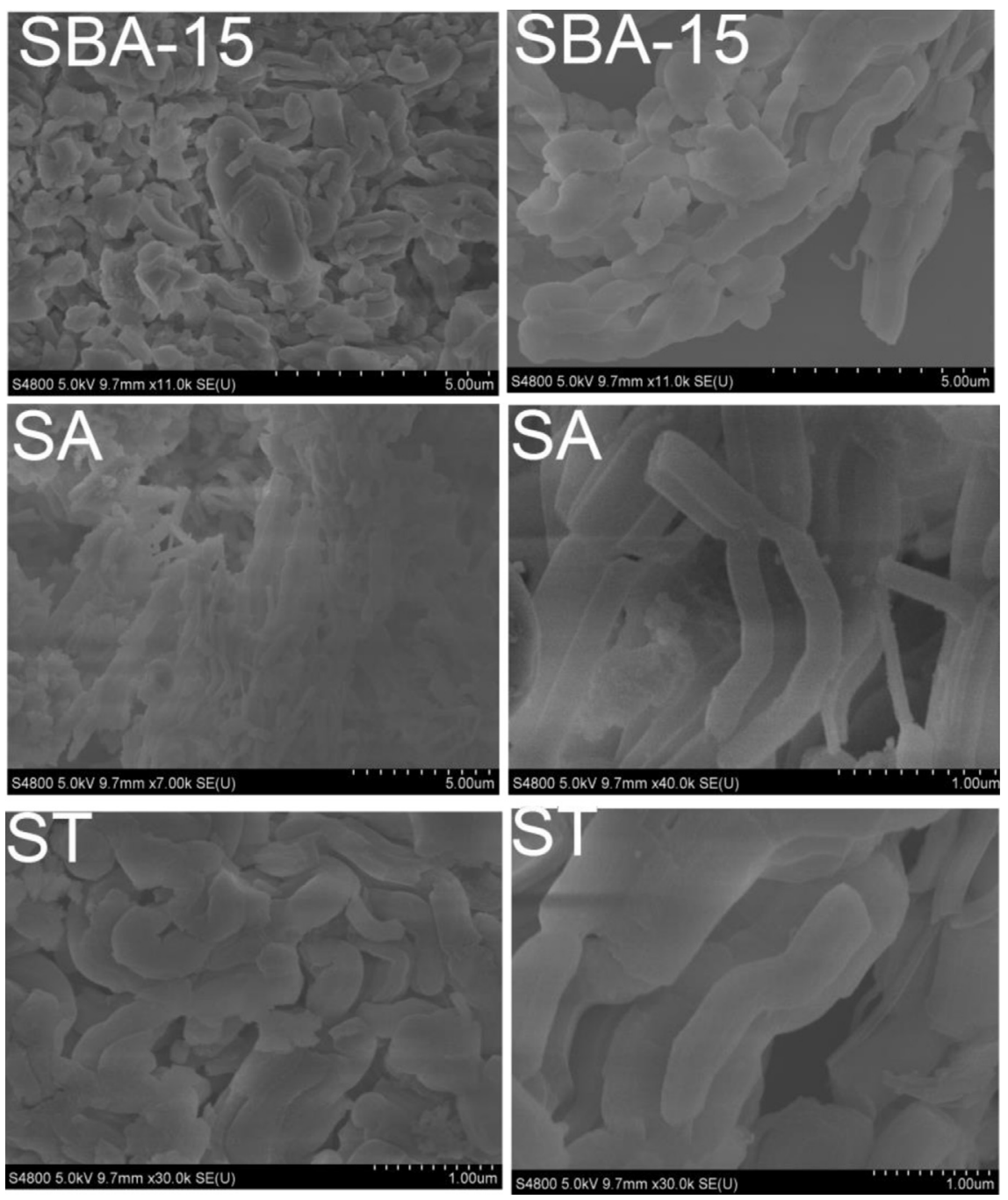

Fig. 3. SEM images SBA-15 (above), SA (middle), and ST (below) mesoporous silica at resolutions of 5 and $1 \mu \mathrm{m}$.

charged amine group of SA and the anion of PCP species as well as the filling of SBA-15 mesopores by charged PCP species in solution are responsible for PCP removal from solution. The efficiency of the electrostatic interaction as a PCP removal mechanism is confirmed by the $\geq 82 \%$ increase in adsorption upon amine functionalization. PCP removal via filling of SBA-15 mesopores might be implicated as a significant removal mechanism because functionalization attaches dangling amino-propyl-triethoxy-silane groups on the surface of SBA-15 mesopores (Fig. 1b), and this acts as obstacle to the free movement of trapped PCP species within the mesopores into the bulk solution, thus enhancing PCP removal from solution by pore filling/trapping on these mesopores. PCP sorption by ST showed $\approx 76 \%$ increment in sorption when compared to the SBA15. The proposed structural interactions of NaTPP with SBA-15 are shown in Fig. 1 (c and d). The relatively lower sorption performance of ST in comparison with SA was attributed to reduction in the amounts of the $-\mathrm{NH}_{3}^{+}$groups in $\mathrm{ST}$ resulting from interaction of the $-\mathrm{NH}_{3}^{+}$groups with the tripolyphosphate ions. This interaction reduces the available- $\mathrm{NH}_{3}^{+}$, but increases the mesh-like nature of the pores hence the ability of the PCP species to be trapped in the pores. However, due to the better efficiency of electrostatic interactions for removal of PCP, SA having more $-\mathrm{NH}_{3}^{+}$groups showed higher sorption capacity than ST at the prevailing experimental conditions.

\subsection{SBA-15 PCP sorption kinetics}

Sorption data was evaluated using the Lagergren pseudo-first and second order kinetics models as well as the Elovich and IPD kinetics models. The model parameters of all SBA-15 sorbents are shown in Table 2. Comparison of the estimated model sorption capacity $\left(q_{\mathrm{e}}\right)$ and the correlation coefficient $\left(r^{2}\right)$ values of the pseudo-first-order and pseudo-second-order kinetics showed that the PCP sorptions fit the pseudo-second order kinetics model better. The $r^{2}$ values of the pseudo-second-order model (0.998-0.999) were closer to unity than those of the pseudo-first-order model $(0.122-0.372)$ and $q_{\mathrm{e}}$ values were also better correlated with the experimental sorption values. This is an indication that the sorption of PCP by these SBA-15 sorbents involves chemisorption occurring by sharing or exchange of electrons between the SBA-15 materials and the anionic PCP species in solution (Olu-Owolabi et al., 2012).

The Elovich and intra-particle diffusion kinetics models gave further insight into PCP sorption mechanism. The estimated Elovich model sorption capacity parameter $-q_{\mathrm{e}}$ values (Table 2 ) suggested that PCP sorption may significantly be explained by this model. The model plot depicted in Fig. 4b implies that for PCP sorption on these SBA-15 materials, there is some degree of boundary layer control between the solution PCP species and the SBA-15 surface active sorption sites. This boundary layer control is assumed to be related 

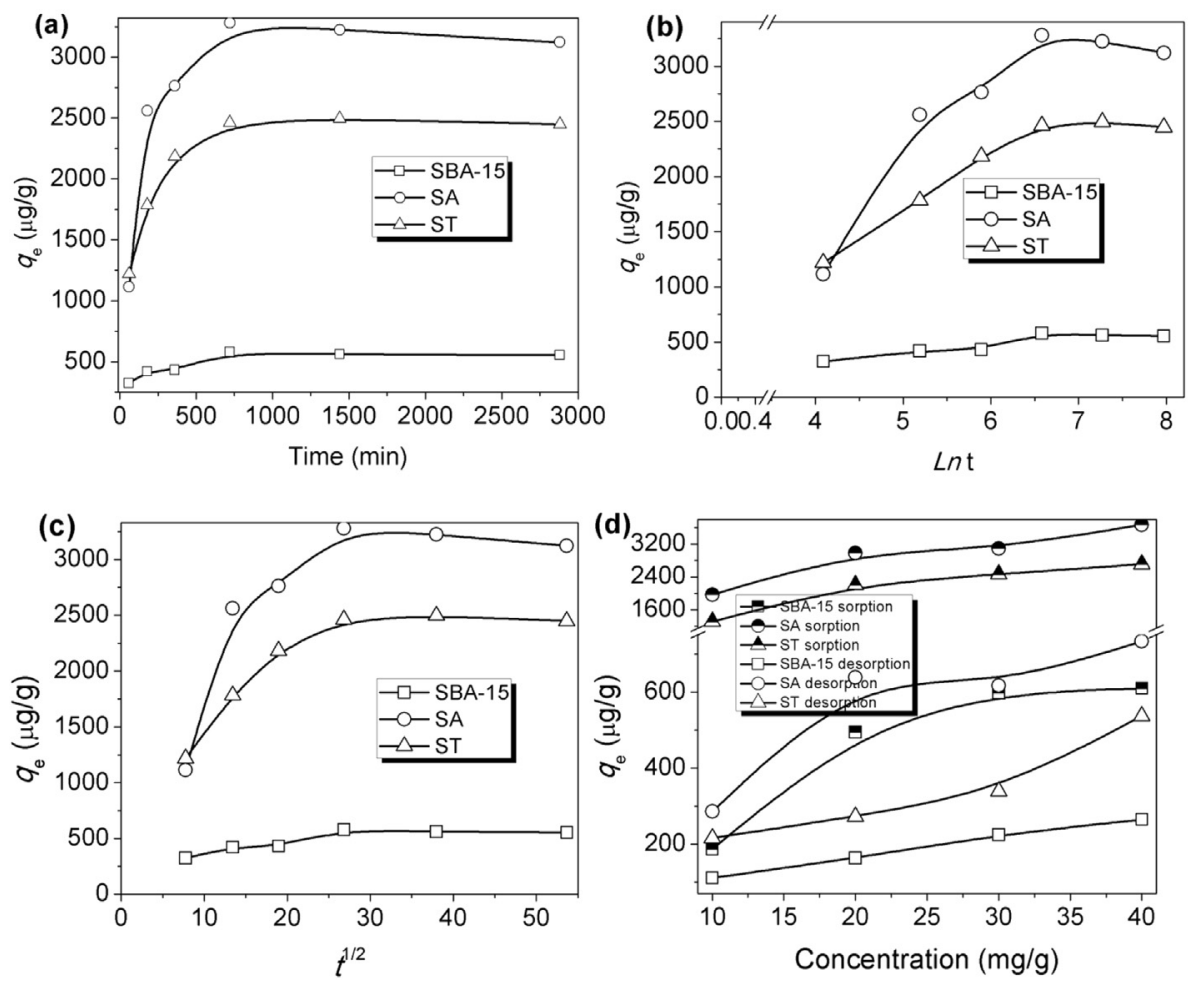

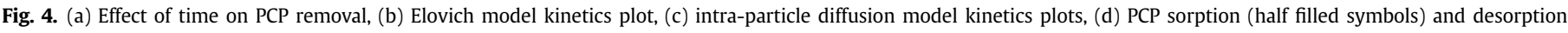
(unfilled symbols) trends at varying solution PCP concentrations.

to the rate controlling mechanism and involves electrostatic interactions.

The IPD kinetics model parameters and plot are shown in Table 2 and Fig. 4c, respectively. These parameters were employed in predicting the sorption mechanism. The plot showed two portions: an initial linear but steep and rapid portion, and a later horizontal portion. Since the linear plot of $q_{\mathrm{t}}$ versus $t^{1 / 2}$ is about $75 \%$ linear at the initial sorption stages, intra-particle diffusion was the main sorption mechanism in this part of the plot. The later portions of the plot were slow and controlled by equilibrium diffusion mechanism which occurred when the rate of the sorptions and desorptions are insignificant. The values of IPD model parameter, $C\left(\mu \mathrm{g} \mathrm{g}^{-1}\right)$, indicated the thickness of the sorbed PCP species at the boundary layer; and it implies that since the values are about $40 \%$ less than the sorption capacity of the corresponding SBA-15 materials, sorptions of PCP are on sorption sites other than those on the surfaces such those within the mesopores.

\subsection{Equilibrium sorption of PCP on pristine and functionalized SBA-} 15

Another important method for describing a sorbent is through evaluation of the adsorption isotherm (Olu-Owolabi et al., 2014). Fig. 4d showed that PCP sorption (half filled symbols) on these<smiles>[O-]c1c(Cl)c(Cl)c(Cl)c(Cl)c1Cl</smiles>

Fig. 5. Unionized PCP in acidic pH medium (left) and ionized PCP in neutral/basic pH medium (right). sorbents increase with PCP solution concentration until equilibrium is reached where no further significant sorption is recorded. The sorption trend is SA > ST > SBA-15. The higher sorption capacity of SA was attributed to the presence of $-\mathrm{NH}_{3}^{+}$groups on both the surface and the pores which are available for electrostatic interactions with the anionic PCP species.

Desorption experiments results (Fig. $4 \mathrm{~d}$ - unfilled symbols) showed that SBA-15 exhibited the least desorption hysteresis. This ease of desorption of PCP by SBA-15 can be related to the earlier proposed $\mathrm{PCP}$ sorption mechanism: that insignificant forces hold PCP species onto the surfaces of SBA-15; thus shaking (at $100 \mathrm{rpm}$ ) of the SBA-15 with the adsorbed PCP can result in up to $50 \%$ desorption. Hysteresis was significantly higher for SA and ST mesoporous silica. Desorptions of $\approx 15$ and $20 \%$, respectively, were observed for SA and ST. These observations were attributed to the charge and nature of the PCP sorption forces: the electrostatic

\section{Table 2}

Kinetics model parameters for PCP sorption on SBA-15, SA, and ST.

\begin{tabular}{lllll}
\hline Kinetics model & Model parameters & \multicolumn{2}{l}{ SBA-15 samples } \\
\cline { 3 - 5 } & & SBA-15 & SA & ST \\
\hline pseudo-first-order & $q_{\mathrm{e}}\left(\mu \mathrm{g} \mathrm{g}^{-1}\right)$ & 73.45 & 468.81 & 366.44 \\
& $K_{1}\left(\mathrm{~min}^{-1}\right)$ & 0.001 & 0.001 & 0.001 \\
& $r^{2}$ & 0.122 & 0.184 & 0.372 \\
pseudo-second-order & $q_{\mathrm{e}}\left(\mu \mathrm{g} \mathrm{g}^{-1}\right)$ & 568.18 & 3225.81 & 2500.00 \\
& $K_{2}\left({\left.\mathrm{~g} \mu \mathrm{g}^{-1} \mathrm{~min}^{-1}\right)}\right.$ & $3.54 \mathrm{E}^{-05}$ & $6.46 \mathrm{E}^{-06}$ & $8.45 \mathrm{E}^{-06}$ \\
Elovich & $r^{2}$ & 0.998 & 0.997 & 0.999 \\
& $q_{\mathrm{e}}\left(\mu \mathrm{g} \mathrm{g}^{-1}\right)$ & 551.70 & 3232.17 & 2468.50 \\
& $\beta$ & 0.015 & 0.002 & 0.003 \\
Intra-particle diffusion & $R^{2}$ & 0.835 & 0.750 & 0.858 \\
& $K_{\mathrm{id}}\left(\mu \mathrm{g} \mathrm{g}^{-1}\right)$ & 350.40 & 1787.00 & 1476.00 \\
& $r^{2}$ & 4.85 & 33.66 & 23.56 \\
Experiment & $q_{\mathrm{e}}\left(\mu \mathrm{g} \mathrm{g}^{-1}\right)$ & 0.655 & 0.493 & 0.622 \\
\hline & $q_{\mathrm{e}}\left(\mu \mathrm{g} \mathrm{g}^{-1}\right)$ & 534.37 & 3064.31 & 2370.04 \\
& & 579.84 & 3279.59 & 2462.82 \\
\hline
\end{tabular}


interaction holding the PCP species in place as well as the mesh-like nature of the mesopores act in tandem to hold back the PCP species within these mesoporous materials, hence the reduced desorption and hysteresis. The observed desorption trend is SBA-15 $>$ ST $>$ SA.

\subsection{Adsorption isotherm models}

The equilibrium sorption data of the PCP on SBA-15, SA, and ST were fitted to two different isotherm models - Langmuir and Freundlich adsorption models, and values of the isotherm parameters for both models are shown in Table 3. The isotherm plots of PCP sorption onto SBA-15 materials were all non-linear as evidenced in the small $n$ values of less than 1 (ranging from 0.29 to 0.87 ) - Table 3. Small $n$ values represent sorption on predominantly heterogeneous sorption sites and since these $n$ values decreased with increasing sorption capacity, it may be regarded as an index of site energy distribution (Olu-Owolabi et al., 2014; Weber et al., 1992). In the case of PCP sorption on these SBA-15 sorbents, part of the PCP species in solution has been partitioned by filling the empty or mesh-like pores of the SBA-15 sorbents, while the bulk has been sorbed by a more specific electrostatic interaction between the PCP anionic species and the cationic amine groups of the sorbents to give an $n$ of less than 1. SBA-15 and its functionalized derivatives are high surface area mesoporous materials, and Nguyen et al. (2007) have reported that adsorptive pore-filling is an important sorption mechanism for porous materials. Hence, two processes are involved in the sorption of PCP species from solution: adsorptive pore-filling and electrostatic interactions.

Comparing the $q_{e}\left(\mu \mathrm{g} \mathrm{g}^{-1}\right)$ and $r^{2}$ values showed that PCP sorption data fit the Freundlich isotherm better than the Langmuir. The regression coefficients $\left(r^{2}\right)$ values of the Freundlich isotherm were closer to unity than the Langmuir and the $q_{e}$ values calculated from the model were also better correlated with the experimental $q_{e}$ values than those of the Langmuir isotherm. The Langmuir isotherm model assumes that the adsorption sites possess equal affinity for the species, and a monolayer is formed at saturation of these sorbent surface sorption sites; the Freundlich isotherm, however, assumes that the adsorption is on heterogeneous surface sites and multi-layer of the sorbate is formed at surface sites at saturation. The observed fit of the equilibrium data to the Freundlich isotherm is in line with the earlier predicted mechanism of PCP sorptions on the SBA-15 materials' that sorption is via adsorptive pore-filling and electrostatic interactions.

One potential application of SA and ST may be in soil amendments to immobilize pesticides; the immobilized pesticide can then be manipulated for controlled pesticide release, hence reducing the continuous use of such chemicals and the consequent pollution of surface and aquifer waters. Further investigations are in progress on the use of functionalized SBA-15 for pesticide immobilization.

\section{Conclusion}

In this study, SBA-15, amine functionalized SBA-15 (SA), and tripolyphosphate functionalized SBA-15 (ST) were synthesized.

Table 3

PCP sorption models parameters for the Langmuir and Freundlich models.

\begin{tabular}{|c|c|c|c|c|c|c|c|}
\hline & \multicolumn{3}{|c|}{ Langmuir sorption model } & \multicolumn{4}{|c|}{ Freundlich sorption model } \\
\hline & $q_{\mathrm{e}}\left(\mu \mathrm{g} \mathrm{g}^{-1}\right)$ & $b$ & $r^{2}$ & $q_{\mathrm{e}}\left(\mu \mathrm{g} \mathrm{g}^{-1}\right)$ & $n$ & $K_{\mathrm{f}}$ & $r^{2}$ \\
\hline SBA-15 & 2083.33 & 0.01 & 0.302 & 720.01 & 0.87 & 31.99 & 0.867 \\
\hline SA & 4166.67 & 0.23 & 0.980 & 3626.05 & 0.29 & 1383.57 & 0.908 \\
\hline ST & 3448.28 & 0.12 & 0.987 & 2855.02 & 0.43 & 663.74 & 0.906 \\
\hline
\end{tabular}

These were used for the removal of PCP from aqueous solution. SBA-15 was not efficient for the PCP removal due to its low sorption and high desorption. SA and ST showed marked improvements for PCP sorption and higher desorption hysteresis. Two processes were implicated in the sorption of PCP species from solution: adsorptive pore-filling of the empty mesopores and electrostatic interactions between charged surface groups on the functionalized SBA-15 mesostructure and the anions of PCP species in solution. Results of this study suggest that functionalized SBA-15 may be applicable for removal of PCP from aqueous phase especially in wastewater treatment.

\section{Acknowledgments}

We also acknowledge the supports of the World Academy of Sciences (TWAS), Trieste Italy and the Chinese Academy of Sciences, China for the award of CAS-TWAS Postgraduate Fellowship (FR number: 3240255024) to P.N. Diagboya; Chief S.L. Edu/Chevron Research grant Nigeria, late Mrs. Rebecca A. Okoh, and Mr. Victor P.O. Okoh, Department Estate Management, School of Environmental Sciences, Yaba College of Technology, Lagos Nigeria.

\section{Appendix A. Supplementary data}

Supplementary data related to this article can be found at http:// dx.doi.org/10.1016/j.jenvman.2014.04.038.

\section{References}

Abdel-Salam, M., Burk, R.C., 2009. Thermodynamics and kinetics studies of pentachlorophenol adsorption from aqueous solutions by multi-walled carbon nanotubes. Water Air Soil. Pollut. 210, 101-111.

Bernardoni, F., Fadeev, A.Y., 2011. Adsorption and wetting characterization of hydrophobic SBA-15 silicas. J. Colloid Interf. Sci. 356, 690-698.

Bruzzoniti, M.C., Prelle, A., Sarzanini, C., Onida, B., Fiorilli, S., Garrone, E., 2007 Retention of heavy metal ions on SBA-15 mesoporous silica functionalised with carboxylic groups. J. Sep. Sci. 30, 2414-2420.

Bui, T.X., Kang, S.Y., Lee, S.H., Choi, H., 2011. Organically functionalized mesoporous SBA-15 as sorbents for removal of selected pharmaceuticals from water J. Hazard. Mater. 193, 156-163.

Calleja, G., Sanz, R., Arencibia, A., Sanz-Pérez, E.S., 2011. Influence of drying conditions on amine-functionalized SBA-15 as adsorbent of $\mathrm{CO}_{2}$. Top. Catal. 54, 135-145.

Choi, J.W., Lee, S.Y., Lee, S.H., Lee, K.B., Kim, D.J., Hong, S.W., 2011. Adsorption of phosphate by amino-functionalized and co-condensed SBA-15. Water Air Soil. Pollut. 223, 2551-2562.

Corriu, R.J.P., Mehdi, A., Rey, C., Thieuleux, C., Frenkel, A., Gibaud, A., 2004. Preparation of ordered SBA-15 mesoporous silica containing chelating groups. Study of the complexation of Euiii inside the pore channels of the materials. New. J. Chem. 28, 156.

Dou, B., Hu, Q., Li, J., Qiao, S., Hao, Z., 2011. Adsorption performance of VOCs in ordered mesoporous silicas with different pore structures and surface chemistry. J. Hazard. Mater. 186, 1615-1624.

Eisler, R., 1989. Pentachlorophenol Hazards to Fish, Wildlife, and Invertebrates: a Synoptic Review. Contam. Hazard. Rev. Report No. 17.

EPA, 1980. Ambient Water Quality Criteria for Pentachlorophenol. U.S. Environ. Protection Agency Rep. 440/5-80-065; p. 89.

Fattori, N., Maroneze, C.M., da Costa, L.P., Strauss, M., Sigoli, F.A., Mazali, I.O. Gushikem, Y., 2012. Ion-exchange properties of imidazolium-grafted SBA-15 toward $\mathrm{AuCl}_{4}(-)$ anions and their conversion into supported gold nanoparticles. Langmuir 28, 10281-10288.

Fisher, B., 1991. Pentachlorophenol: Toxicology and environmental fate. J. Pest. Reform. 11, 2-5.

Freundlich, H.M.F., 1906. Über die adsorption in lösungen. Z. für Phys. Chem. 57A, $385-470$.

Katiyar, A., Yadav, S., Smirniotis, P.G., Pinto, N.G., 2006. Synthesis of ordered large pore SBA-15 spherical particles for adsorption of biomolecules. J. Chromatogr. A. 1122, 13-20.

Lagergren, S., 1898. Zur theorie der sogenannten adsorption gelöster stoffe. kungliga svenska vetenskapsakademiens. Handlingar 24, 1-39.

Langmuir, I., 1916. The constitution and fundamental properties of solids and liquids. J. Amer. Chem. Soc. 38, 2221-2295.

Laus, R., Costa, T.G., Szpoganicz, B., Favere, V.T., 2010. Adsorption and desorption of $\mathrm{Cu}(\mathrm{II}), \mathrm{Cd}(\mathrm{II})$ and $\mathrm{Pb}(\mathrm{II})$ ions using chitosan crosslinked with epichlorohydrintriphosphate as the adsorbent. J. Hazard. Mater. 183, 233-241. 
Lee, S.T., Mi, F.L., Shen, Y.J., Shyu, S.S., 2001. Equilibrium and kinetic studies of copper(II) ion uptake by chitosan-tripolyphosphate chelating resin. Polymer 42, 1879-1892.

Li, C., Yang, J., Shi, X., Liu, J., Yang, Q., 2007. Synthesis of SBA-15 type mesoporous organosilicas with diethylenebenzene in the framework and post-synthetic framework modification. Micropor. Mesopor. Mat. 98, 220-226.

Li, R., Wen, B., Zhang, S., Pei, Z., Shan, X., 2009. Influence of organic amendments on the sorption of pentachlorophenol on soils. J. Environ. Sci. 21, 474-480.

Margolese, D., Melero, J.A., Christiansen, S.C., Chmelka, B.F., Stucky, G.D., 2000. Direct syntheses of ordered SBA-15 mesoporous silica containing sulfonic acid groups. Chem. Mater. 2000, 2448-2459.

Momčilović, M.Z., Ranđelović, M.S., Zarubica, A.R., Onjia, A.E., Kokunešoski, M., Matović, B.Z., 2013. SBA-15 templated mesoporous carbons for 2,4-dichlorophenoxyacetic acid removal. Chem. Eng. J. 220, 276-283.

Mureseanu, M., Reiss, A., Stefanescu, I., David, E., Parvulescu, V., Renard, G. Hulea, V., 2008. Modified SBA-15 mesoporous silica for heavy metal ions remediation. Chemosphere 73, 1499-1504.

Nguyen, T.H., Cho, H.H., Poster, D.L., Ball, W.P., 2007. Evidence for a pore-filling mechanism in the adsorption of aromatic hydrocarbons to a natural wood char. Environ. Sci. Technol. 41, 1212-1217.

Olu-Owolabi, B.I., Diagboya, P.N., Adebowale, K.O., 2014. Evaluation of pyrene sorption-desorption on tropical soils. J. Environ. Manage. 137, 1-9.

Olu-Owolabi, B.I., Diagboya, P.N., Ebaddan, W.C., 2012. Mechanism of $\mathrm{Pb}^{2+}$ remova from aqueous solution using a nonliving moss biomass. Chem. Eng. J. 195-196, $270-275$.
Panda, R.N., Hsieh, M.F., Chung, R.J., Chin, T.S., 2003. FTIR, XRD, SEM and solid state NMR investigations of carbonate-containing hydroxyapatite nano-particles synthesized by hydroxide-gel technique. J. Phys. Chem. Solids 64, 193-199.

Parikh, S.J., Chorover, J., 2006. ATR-FTIR spectroscopy reveals bond formation during bacterial adhesion to iron oxide. Langmuir 22, 8492-8500.

Sureshkumar, M.K., Das, D., Mallia, M.B., Gupta, P.C., 2010. Adsorption of uranium from aqueous solution using chitosan-tripolyphosphate (CTPP) beads. J. Hazard. Mater. 184, 65-72.

Thielemann, J.P., Girgsdies, F., Schlögl, R., Hess, C., 2011. Pore structure and surface area of silica SBA-15: influence of washing and scale-up. Beilstein J. Nanotechnol. 2, 110-118.

Wang, X., Lin, K.S., Chan, J.C., Cheng, S., 2004. Preparation of ordered large pore SBA15 silica functionalized with aminopropyl groups through one-pot synthesis. Chem. Commun. (Camb.) 23, 2762-2763.

Weber, W.J.J., McGinley, P.M., Katz, L.E., 1992. A distributed reactivity model for sorption by soils and sediments. 1. conceptual basis and equilibrium assessments. Environ. Sci. Technol. 26, 1955-1962.

Weber, W.J., Morris, J.C., 1963. Kinetics of adsorption on carbon from solutions. J. Sanit. Eng. Div. Amer. Soc. Civ. Eng. 89, 31-60.

Xu, Y.Q. Zhou, G.W., Wu, C.C., Li, T.D., Song, H.B., 2011. Improving adsorption and activation of the lipase immobilized in amino-functionalized ordered mesoporous SBA-15. Solid State Sci. 13, 867-874.

Zhao, D., Feng, J., Huo, O., Melosh, N., Fredrickson, G.H., Chmelka, B.F., Stucky, G.D., 1998. Triblock copolymer syntheses of mesoporous silica with periodic 50 to 300 angstrom pores. Science 279, 548-552. 